\title{
Implantação e efeitos da Estratégia Amamenta Alimenta Brasil nas Unidades de
}

\section{Saúde de Piracicaba/SP}

\author{
Implementation and effects of The Brazilian Breastfeeding Food Strategy in the Health Units of \\ Piracicaba/SP
}

Implementación y efectos de la Estrategia Amamenta Alimenta Brasil en las Unidades de Salud de

Piracicaba/SP

Recebido: 07/10/2021 | Revisado: 16/10/2021 | Aceito: 24/10/2021 | Publicado: 26/10/2021

\author{
Tatiana do Prado Lima Bonini \\ ORCID: https://orcid.org/0000-0002-8958-7084 \\ Universidade Estadual de Campinas, Brasil \\ E-mail: tati.bonini36@gmail.com \\ Carolina Matteussi Lino \\ ORCID: https://orcid.org/0000-0001-6686-3296 \\ Universidade Estadual de Campinas, Brasil \\ E-mail: carolina.matteussi@gmail.com \\ Maria da Luz Rosário de Sousa \\ ORCID: https://orcid.org/0000-0002-0346-5060 \\ Universidade Estadual de Campinas, Brasil \\ E-mail: luzsousa@fop.unicamp.br \\ Marília Jesus Batista de Brito Mota \\ ORCID: https://orcid.org/0000-0002-0379-3742 \\ Universidade Estadual de Campinas, Brasil \\ E-mail: mariliajbatista@yahoo.com.br
}

\begin{abstract}
Resumo
Objetivo: avaliar a implantação e os efeitos observados da Estratégia Amamenta Alimenta Brasil (EAAB) em Unidades de Saúde (USs) de Piracicaba/SP. Métodos: Pesquisa avaliativa realizada em duas etapas: 1) análise da adesão das equipes à EAAB; 2) análise comparativa dos marcadores de consumo alimentar em USs com e sem EAAB. Na etapa 1 os dados foram obtidos por um instrumento de monitoramento das ações pactuadas nas oficinas de implantação da EAAB e, na etapa 2, por marcadores de consumo alimentar registrados no Sistema de Vigilância Alimentar e Nutricional no período de 2015 a 2018. Resultados: Na etapa 1, 100\% das USs tiveram adesão de 80-85\% da equipe e pactuaram ações para a promoção do aleitamento materno e alimentação complementar saudável. Na etapa 2, as US com EAAB apresentaram aumento do percentual médio dos indicadores de aleitamento materno exclusivo em menores de 6 meses, aleitamento materno complementar, introdução alimentar e redução no indicador de consumo de macarrão instantâneo. Conclusão: Houve boa adesão à EAAB por parte dos membros das equipes e os indicadores de promoção do aleitamento materno e alimentação complementar saudável das USs com EAAB foram mais positivos em relação às sem EAAB.

Palavras-chave: Aleitamento materno; Nutrição da criança; Atenção Primária à Saúde; Políticas públicas em saúde; Avaliação em saúde.

Abstract

Objective: to evaluate the implementation and observed effects of the Strategy Breastfeed and Feed Brazil (SBFB) in Health Units (HU) in Piracicaba/SP. Methods: evaluative research carried out in two stages: 1) analysis of teams' adherence to SBFB; 2) comparative analysis of food consumption markers in HUs with and without SBFB. In step 1, data were obtained by an instrument for monitoring the actions agree in the SBFB implementation workshops and, in step 2, by food consumption markers registered in the Food and Nutrition Surveillance System for the period from 2015 to 2018. Results: In step 1, 100\% of the HUs had 80-85\% adherence by the team and agreed on actions to promote breasteeding and healthy complementary feeding. In step 2, HUs with SBFB showed an increase in the average percentage of exclusive breastfeeding indicators in children under 6 months, complementary breastfeeding, introduction of food and a reduction in the instant noodles consumption indicator. Conclusion: There was good adherence to SBFB by the team members and the indicators for promoting of breastfeeding and healthy complementary feeding of the HUs with SBFB were more positive than those without SBFB.
\end{abstract}

Keywords: Breastfeeding; Child nutrition; Primary health care; Public health policy; Health evaluation. 


\begin{abstract}
Resumen
Objetivo: evaluar la implementación y efectos observados de la Estrategia Amamenta Alimenta Brasil (EAAB) en Unidades de Salud (US) en Piracicaba/SP. Métodos: Investigación evaluativa realizada en dos etapas: 1) análisis de la adherencia de los equipos a la EAAB; 2) análisis comparativo de marcadores de consumo de alimentos en US con y sin EAAB. En el paso 1, los datos se obtuvieron mediante un instrumento de seguimiento de las acciones acordadas en los talleres de implementación de la EAAB y, en el paso 2, por los marcadores de consumo de alimentos registrados en el Sistema de Vigilancia Alimentaria y Nutricional en el período de 2015 a 2018. Resultados: En la etapa 1, el $100 \%$ de las US tuvo 80-85\% de adhesión del equipo y acordaron acciones para promover la lactancia materna y la alimentación complementaria saludable. En el paso 2, la US con EAAB mostró un aumento en el porcentaje medio de indicadores de lactancia materna exclusiva en menores de 6 meses, lactancia materna complementaria, introducción de alimentos y una reducción en el indicador de consumo de fideos instantáneos. Conclusión: Hubo una buena adherencia a la EAAB por parte de los miembros del equipo y los indicadores de promoción de la lactancia materna y alimentación complementaria saludable en las US con EAAB fueron más positivos en comparación con aquellos sin EAAB.
\end{abstract}

Palabras clave: Lactancia materna; Nutrición infantil; Primeros auxilios; Políticas de salud pública; Valoración de salud.

\title{
1. Introdução
}

A primeira infância é um período de intenso desenvolvimento da criança, sendo a alimentação primordial para que ele ocorra adequadamente (Lopes et al., 2018). A nutrição adequada na infância, pode determinar o desenvolvimento e a saúde do indivíduo ao longo da vida adulta (Silveira et al., 2008; Cunha et al., 2015) e seus benefícios, nos primeiros mil dias da criança- que contempla aleitamento materno exclusivo (AME), aleitamento materno complementado (AMC) e alimentação complementar saudável (ACS) - incluem a melhora no desenvolvimento cognitivo, motor e social e a diminuição do risco de obesidade e doenças crônicas não transmissíveis. Por este motivo, estratégias que promovam o aleitamento materno (AM) e a melhora da ACS são de grande relevância (Brasil, 2013a).

O leite materno é o alimento completo para a criança em sua fase inicial da vida, na perspectiva nutricional, imunológica e de desenvolvimento, melhorando o Quociente de Inteligência (QI), capacidade educacional e renda, reduzindo a morbidade e mortalidade infantil (Victora et al., 2015; Victora et al, 2016; Alves et al., 2018). Segundo estudo realizado por Walters et al. (2019), não aleitar foi atribuído à ocorrência 166 milhões de casos evitáveis de diarreia e 9 milhões de pneumonia em crianças menores de 2 anos, bem como à perda de 694,322 vidas e de perdas econômicas - somando-se custos com sistema de saúde, custos com a mortalidade e custos com perdas cognitivas - estimadas em \$341,3 bilhões de dólares. Por este motivo, o Ministério da Saúde (MS) preconiza o AME até o sexto mês de vida da criança e AMC até os dois anos ou mais (Brasil, 2015a).

Segundo o relatório "Global Breastfeeding Scorecard”, da World Health Organization [WHO] (2017), que analisa a implementação de ações para o apoio ao AM, apenas $40 \%$ das crianças menores de seis meses foram amamentadas exclusivamente e, apenas 23 países dos 197 avaliados têm taxa de AME acima de 60\%. Países como China e Nigéria, apresentaram prevalência de AME de 21\%, ao passo que Índia, México e Indonésia, 55\%, 31\% e 41\% respectivamente. No Brasil, a prevalência de AME era de $41 \%$ em 2008 enquanto que, em estudo realizado em três municípios da região de Ribeirão Preto, em 2012, o índice foi de 38\% (Brasil, 2009; Venâncio et al., 2016). Apesar da prevalência no Brasil estar abaixo do preconizado pela OMS, ela encontra-se mais alta do que nos países desenvolvidos - que é inferior a $20 \%$-, sendo este indicador considerado como um dos poucos comportamentos positivos encontrados em países em desenvolvimento (Victora et al., 2015). No município de Piracicaba, a prevalência de AME em 2009 encontrava-se inferior à média brasileira $(36,4 \%)$ e, também, ao recomendado pela OMS (90 a 100\%) (Brasil, 2009).

Dados da II Pesquisa de Prevalência de AM apontaram a introdução de comida salgada e frutas para crianças entre o terceiro e sexto mês de vida, além do consumo de alimentos não recomendados para menores de dois anos (como bolachas, salgadinhos, refrigerantes e café) entre crianças de 9 a 12 meses (Brasil, 2009). Estes dados demonstram o desafio de mudar o 
padrão de consumo alimentar em menores de dois anos e a relevância das ações no âmbito da $\mathrm{AB}$, especialmente nas Unidades de Saúde da Família (USFs) (Jaime et al., 2011; Torigoe et al., 2012).

Diante deste cenário, em 2012 o MS lançou a Estratégia Nacional para Promoção do Aleitamento Materno e Alimentação Complementar Saudável - Estratégia Amamenta e Alimenta Brasil (EAAB). Derivada da integração entre a Rede Amamenta Brasil e a Estratégia Nacional para a Alimentação Complementar Saudável, a estratégia tem por objetivo reforçar e incentivar a promoção do AM e da ACS para crianças menores de dois anos de idade no âmbito do Sistema Único de Saúde (Brasil, 2013b). Para atingir esse objetivo, a EAAB prevê, além de oficinas de implantação, reuniões de monitoramento, com o intuito de garantir a incorporação das propostas no âmbito da Atenção Básica (AB) (Brasil, 2013c).

Considerando a necessidade de incentivar as estratégias que auxiliem na melhora da amamentação e alimentação saudável e o monitoramento das práticas de consumo alimentar, o presente estudo teve por objetivo, avaliar a implantação da EAAB nas USs, a partir do monitoramento das ações propostas pelas unidades e os efeitos destas ações nos indicadores de amamentação e alimentação complementar saudável.

\section{Metodologia}

\section{Delineamento do estudo}

Trata-se de uma pesquisa avaliativa do tipo "análise de implantação". A pesquisa avaliativa é capaz de analisar as intervenções e necessidades em saúde, a partir da integração entre saberes e práticas disponíveis com o objetivo de modificar os problemas de saúde da população (Arreaza \& Moraes, 2010).

O estudo foi realizado em em 2 etapas: 1) análise da adesão das equipes à EAAB após as oficinas de implantação e 2) análise comparativa dos marcadores de consumo alimentar em USs com e sem a EAAB.

\section{Local do estudo}

O estudo foi realizado no município de Piracicaba, São Paulo. Segundo o Instituto Brasileiro de Geografia e Estatística (IBGE), em 2017, sua população estimada era de 397.322 habitantes (IBGE, 2017) e, de acordo com o Instituto de Pesquisas e Planejamento de Piracicaba (IPPLAP) seu Índice de Desenvolvimento Humano Municipal (IDHM), em 2010, é 0,785, considerado alto na classificação do Programa das Nações Unidas para o Desenvolvimento (IPPLAP, 2017). Atualmente o município possui 71 USs, sendo 51 USFs, nove Centros de Referência em Atenção Básica (CRABs) e 11 Unidades Básicas de Saúde (UBSs) (IPPLAP, 2017).

O município iniciou suas atividades para a implantação da EAAB em novembro/2014, a partir de um treinamento para formação de tutores. Em um primeiro momento, cinco USs foram selecionadas para receber as equipes de tutores para a realização da oficina de trabalho e, a partir de março/2015, Piracicaba extendeu o processo de implantação para as demais USs, priorizando unidades participantes do Programa de Melhoria do Acesso e da Qualidade na Atenção Básica (PMAQ-AB). As oficinas de implantação tinham duração de 4 horas ininterruptas, visando uma execução eficiente e esclarecedora das atividades, e foram desenvolvidas seguindo os passos propostos pelo MS. As unidades que cumpriram os critérios de implementação instituídos pelo MS receberam certificação da EAAB.

\section{Variáveis do estudo}

As variáveis utilizadas para a análise da implantação da EAAB - etapa 1 - foram baseadas nos critérios de implementação, sendo: 1) participação da equipe de saúde (80 a 85\%) nas oficinas de implantação da EAAB; 2) monitoramento das ações sistemáticas pactuadas, individuais ou coletivas, para a promoção do AM e ACS; 3) disponibilidade de instrumento para detectar problemas relacionados ao AM e ACS, incluindo o preenchimento do Marcador de Consumo 
Alimentar; 4) ter executado, como rotina de trabalho, pelo menos uma ação de incentivo ao AM e uma de ACS dentre as pactuadas no plano de ação. De forma a facilitar a interpretação destas variáveis, as ações estabelecidas no critério 2 foram agrupados por linha de cuidado: orientação de AM, preenchimento do marcador de consumo alimentar, orientação sobre saúde da criança, orientação sobre ACS e orientações na visita domiciliar.

Para a análise dos marcadores de consumo alimentar, adotou-se como variáveis os indicadores: aleitamento materno exclusivo em menores de 6 meses; aleitamento materno continuado; introdução de alimentos; consumo de bebidas adoçadas, macarrão instantâneo, salgadinhos de pacote ou biscoitos salgados e recheados, doces ou guloseimas.

\section{Tamanho e seleção da amostra}

Na etapa 1, a amostra foi composta, por 19 USs que, a partir de 2015, participaram das oficinas de implantação da EAAB e pactuaram ações para o atendimento aos critérios da estratégia.

Na etapa 2, a amostra foi composta por 74 US, sendo 24 unidades que possuíam a EAAB implantada e 50 que não possuiam a EAAB implantada. Todas as unidades que compuseram esta etapa do estudo preencheram, via Sistema de Vigilância Alimentar e Nutricional (SISVAN), o marcador de consumo alimentar de crianças de 0 a 2 anos de idade entre o período de $1^{\circ}$ de janeiro de 2015 a 31 de dezembro de 2018. Ressalta-se que dentre as US sem a EAAB, duas unidades compuseram a amostra por ser uma unidade mista e uma unidade de atenção secundária especializada no atendimento ao adolescente que também realizam atendimento à crianças de 0 a 2 anos de idade.

\section{Instrumento de coleta de dados}

Durante a etapa 1, utilizou-se um instrumento adaptado pelo município de Piracicaba para o monitoramento das ações pactuadas nas oficinas de implantação da EAAB. Este instrumento era composto por questões relacionadas aos critérios de implantação da EAAB que envolviam: participação da equipe nas oficinas, monitoramento de ações para promoção do AM e ACS, preenchimento dos marcadores de consumo alimentar.

Para a etapa 2 não foi necessária a utilização de instrumentos de coleta, uma vez que os dados foram obtidos de forma compilada, diretamente do SIVAN. O SISVAN tem como objetivo gerir e divulgar informações sobre os fatores que influenciam as condições e o estado nutricional da população (a partir de índices antropométricos e dos marcadores de consumo alimentar) (Brasil, 2015), não possibilitando identificação dos indivíduos nesses dados.

\section{Coleta de dados}

Na etapa 1, os dados foram obtidos no período de julho a novembro/2018, durante as reuniões de equipe das USs. Para a avaliação das ações pactuadas pelas equipes nas oficinas, a pesquisadora preenchia o instrumento adaptado pelo município. Ainda nesta etapa, a avaliação do cumprimento das ações pactuadas foi realizada a partir do relato das equipes das US neste encontro com a pesquisadora.

Na etapa 2, os dados foram obtidos a partir de planilhas com os marcadores de consumo alimentar de crianças de 0 a 2 anos por US. Foram obtidos os marcadores de consumo realizados no período de $1^{\circ}$ de janeiro de 2015 a 31 de dezembro de 2018. Ressalta-se que a pesquisadora não tinha acesso aos marcadores de consumo alimentar individuais e que os dados foram disponibilizados de forma agrupada por unidade de saúde.

\section{Análise dos dados}

Para a análise da adesão das equipes à EAAB, realizou-se análise descritiva com frequência e percentual das respostas obtidas via instrumento de monitoramento, ao passo que para a análise dos marcadores de consumo alimentar,utilizou-se as 
fórmulas preconizadas pelo Ministério da Saúde (Brasil, 2015), sendo os resultados apresentados em percentuais médios, considerando sempre o dia anterior a avaliação:

1-Aleitamento Exclusivo em menores de 6 meses: Proporção (\%) de crianças até 5 meses e 29 dias que receberam exclusivamente leite materno.

2- Aleitamento materno continuado: Proporção (\%) de crianças de 6 a 23 meses e 29 dias que receberam leite materno.

3- Introdução de alimentos: Proporção (\%) de crianças de 6 a 8 meses e 29 dias que receberam alimentos na frequência recomendada para a idade. Para crianças de 6 a 6 meses e 29 dias considerar o consumo de duas frutas e uma comida de sal por dia. Para crianças de 7 a 8 meses e 29 dias, o consumo de duas frutas e duas comidas de sal por dia, além do AMC.

4- Consumo de bebidas adoçadas, macarrão instantâneo, salgadinhos de pacote ou biscoitos salgados e recheados, doces ou guloseimas: Proporção (\%) de crianças de 6 a 23 meses e 29 dias que consumiram estes alimentos.

Ainda nesta etapa, após o cálculo dos percentuais médios e desvio padrão dos indicadores realizou-se o teste não paramétrico de Mann Whitney para comparação do AME, AMC, introdução e consumo alimentos entre as USs com e sem a EAAB, com nível de significância de 5\%. As análises foram realizadas no Statistical Package for the Social Sciences (SPSS).

\section{Aspectos éticos}

A pesquisa foi aprovada pelo Comitê de Ética em Pesquisa (protocolo CAAE: 86849418.6.0000.5418) e, em seguida, pela Secretaria de Saúde municipal. O estudo foi conduzido de acordo com todas as diretrizes da Resolução 466/2012.

\section{Resultados}

Durante a etapa 1, foi possível identificar a adesão de 100\% das USs aos critérios 1 - Participação de, no mínimo, 8085\% da equipe nas oficinas de implantação da Estratégia e 2 - Elaboração de ações sistemáticas individuais e coletivas para a promoção do AME e ACS, ao passo que os critérios 3 - Preenchimento do marcador de consumo alimentar e 4 - Ações de incentivo e monitoramento do AM e ACS, foram cumpridos parcial ou totalmente por grande parte das USs (Tabela 1).

Tabela 1 - Frequência e porcentagem das ações avaliadas no monitoramento da EAAB nas USs, segundo critérios do Ministério da Saúde (MS), Piracicaba, 2018 ( $\mathrm{n}=19)$.

\begin{tabular}{|c|c|c|c|}
\hline Variáveis & & $\mathrm{n}$ & $\%$ \\
\hline \multirow{3}{*}{$\begin{array}{l}\text { Critério } 1 \text { - Participação de } 80 \text { a } 85 \% \text { da equipe nas oficinas de implantação } \\
\qquad \text { da EAAB }\end{array}$} & Sim & 19 & 100,0 \\
\hline & Parcial & 0 & 0,0 \\
\hline & Não & 0 & 0,0 \\
\hline \multirow{3}{*}{$\begin{array}{c}\text { Critério } 2 \text { - Ações sistemáticas individuais e coletivas desenvolvidas pela } \\
\text { equipe para a promoção do AME e ACS }\end{array}$} & Sim & 19 & 100,0 \\
\hline & Parcial & 0 & 0,0 \\
\hline & Não & 0 & 0,0 \\
\hline \multirow{3}{*}{ Critério 3 - Instrumento para detectar problemas relacionados ao AM e ACS } & Sim & 12 & 63,2 \\
\hline & Parcial & 6 & 31,6 \\
\hline & Não & 1 & 5,3 \\
\hline \multirow{3}{*}{ Critério 4 - Ação de incentivo ao aleitamento materno e de ACS } & Sim & 15 & 78,9 \\
\hline & Parcial & 4 & 21,1 \\
\hline & Não & 0 & 0,0 \\
\hline
\end{tabular}

Nota: EAAB: Estratégia Amamenta Alimenta Brasil; AME: Aleitamento Materno Exclusivo; AM: Aleitamento Materno; ACS: Alimentação complementar Saudável.

Fonte: Autores. 
O monitoramento das ações propostas durante a implantação da EAAB mostrou que a orientação sobre AM esteve presente em grande parte das USs $(78,9 \%)$ e que o preenchimento dos marcadores de consumo alimentar foi realizado pela maioria das equipes, mesmo que parcialmente. Identificou-se também o cumprimento das ações relacionadas à orientação sobre saúde da criança $(89,5 \%)$, orientação sobre ACS $(89,5 \%)$ e realização de visita domiciliar para orientação sobre AM e ACS $(68,4 \%)$ por grande parte das USs (Tabela 2$)$.

Tabela 2 - Frequência e porcentagem do monitoramento das ações propostas pelas USs na implantação da EAAB, Piracicaba, $2018(n=19)$.

\begin{tabular}{|c|c|c|c|}
\hline Variáveis & & $\mathbf{n}$ & $\%$ \\
\hline \multirow{3}{*}{ Orientação sobre AM } & $\overline{\text { Sim }}$ & 15 & 78,9 \\
\hline & Parcial & 3 & 15,8 \\
\hline & Não & 1 & 5,3 \\
\hline \multirow{3}{*}{ Preenchimento marcador de consumo alimentar } & Sim & 13 & 68,4 \\
\hline & Parcial & 5 & 26,3 \\
\hline & Não & 1 & 5,3 \\
\hline \multirow{3}{*}{ Orientação sobre saúde da criança } & Sim & 17 & 89,5 \\
\hline & Parcial & 0 & 0,0 \\
\hline & Não & 2 & 10,5 \\
\hline \multirow{3}{*}{ Orientação sobre ACS } & Sim & 17 & 89,5 \\
\hline & Parcial & 0 & 0,0 \\
\hline & Não & 2 & 10,5 \\
\hline \multirow{3}{*}{ Visita domiciliar para orientação AM e ACS } & Sim & 13 & 68,4 \\
\hline & Parcial & 2 & 10,5 \\
\hline & Não & 4 & 21,1 \\
\hline
\end{tabular}

Nota: AM: Aleitamento Materno; ACS: Alimentação complementar Saudável. Fonte: Autores.

Da comparação dos indicadores entre USs com e sem a EAAB ao longo do periodo de 2015 a 2018, verificou-se que houve diferença favorável ao grupo com EAAB para a AME no ano de 2018 (p=0,002), para a AMC e introdução de alimentos nos anos de 2017 (p<0,001) e 2018 (p=0,048) e menor consumo de macarrão instantâneo, salgadinhos de pacote ou biscoitos salgados em 2015 ( $\mathrm{p}=0,029)$ e 2017 ( $\mathrm{p}=0,021$ ). Entretanto houve maior prevalência de consumo de biscoito recheado, doces ou guloseimas nas USs com EAAB no ano de 2017 ( $\mathrm{p}=0,004)$ (Tabela 3). 
Tabela 3 - Comparação do percentual médio dos indicadores de AM e ACS da ficha de marcadores de consumo alimentar entre as unidades com e sem a EAAB, SISVAN, Piracicaba, $2018(\mathrm{n}=74)$.

\begin{tabular}{|c|c|c|c|c|c|c|c|c|c|c|}
\hline \multirow{2}{*}{\multicolumn{2}{|c|}{ Indicadores }} & \multicolumn{2}{|c|}{2015} & \multicolumn{2}{|c|}{2016} & \multicolumn{2}{|c|}{2017} & \multicolumn{2}{|c|}{2018} & \multirow[b]{2}{*}{ Total } \\
\hline & & \multirow{2}{*}{$\begin{array}{c}\begin{array}{c}\% \\
\text { Médio }\end{array} \\
4,58\end{array}$} & DP & $\begin{array}{c}\% \\
\text { Médio } \\
\end{array}$ & DP & $\begin{array}{c}\% \\
\text { Médio } \\
\end{array}$ & DP & $\begin{array}{c}\% \\
\text { Médio } \\
\end{array}$ & & \\
\hline \multirow{3}{*}{ AME } & Com EAAB & & $\pm 13,75$ & 17,07 & $\pm 29,06$ & 20,13 & $\pm 16,64$ & 43,60 & $\pm 32,67$ & 24 \\
\hline & Sem EAAB & 2,80 & $\pm 15,12$ & 7,64 & $\pm 23,37$ & 23,92 & $\pm 35,17$ & 19,70 & $\pm 33,60$ & 50 \\
\hline & valor de $\mathrm{p}$ & \multicolumn{2}{|c|}{0,193} & \multicolumn{2}{|c|}{0,088} & \multicolumn{2}{|c|}{0,283} & \multicolumn{2}{|c|}{$0,002 * *$} & \\
\hline \multirow{3}{*}{$\mathrm{AMC}$} & Com EAAB & 26,66 & $\pm 29,07$ & 37,92 & $\pm 29,44$ & 53,79 & $\pm 21,60$ & 56,76 & $\pm 26,43$ & 24 \\
\hline & Sem EAAB & 26,53 & $\pm 34,28$ & 34,83 & $\pm 34,16$ & 18,59 & $\pm 29,83$ & 39,90 & $\pm 38,99$ & 50 \\
\hline & valor de $\mathrm{p}$ & \multicolumn{2}{|c|}{0,698} & \multicolumn{2}{|c|}{0,501} & \multicolumn{2}{|c|}{$0,000 * *$} & \multicolumn{2}{|c|}{$0,048 * *$} & \\
\hline \multirow{3}{*}{$\begin{array}{l}\text { Introdução de } \\
\text { alimentos }\end{array}$} & Com EAAB & 10,94 & $\pm 23,88$ & 20,04 & $\pm 34,03$ & 25,09 & $\pm 29,89$ & 18,40 & $\pm 20,98$ & 24 \\
\hline & Sem EAAB & 7,92 & $\pm 23,66$ & 13,33 & $\pm 29,93$ & 5,66 & $\pm 17,26$ & 9,16 & $\pm 20,45$ & 50 \\
\hline & valor de $\mathrm{p}$ & \multicolumn{2}{|c|}{0,208} & \multicolumn{2}{|c|}{0,168} & \multicolumn{2}{|c|}{$0,000 * *$} & \multicolumn{2}{|c|}{$0,008 * *$} & \\
\hline \multirow{3}{*}{$\begin{array}{c}\text { Consumo de bebidas } \\
\text { adoçadas }\end{array}$} & Com EAAB & 38,85 & $\pm 37,46$ & 20,91 & $\pm 31,77$ & 17,70 & $\pm 17,05$ & 24,74 & $\pm 22,72$ & 24 \\
\hline & Sem EAAB & 44,49 & $\pm 112,98$ & 27,16 & $\pm 37,61$ & 24,11 & $\pm 35,08$ & 21,31 & $\pm 28,93$ & 50 \\
\hline & valor de $\mathrm{p}$ & \multicolumn{2}{|c|}{0,273} & \multicolumn{2}{|c|}{0,826} & \multicolumn{2}{|c|}{0,574} & \multicolumn{2}{|c|}{0,149} & \\
\hline Consumo de macarrão & Com EAAB & 26,74 & $\pm 30,59$ & 27,55 & $\pm 24,77$ & 13,46 & $\pm 13,04$ & 18,40 & $\pm 23,33$ & 24 \\
\hline instantâneo, & Sem EAAB & 17,21 & $\pm 32,07$ & 21,32 & $\pm 30,78$ & 15,82 & $\pm 30,79$ & 14,18 & $\pm 21,76$ & 50 \\
\hline $\begin{array}{l}\text { salgadinhos de pacote } \\
\text { ou biscoitos salgados }\end{array}$ & valor de $\mathrm{p}$ & & & & & & & & & \\
\hline Consumo de biscoito & Com EAAB & 19,24 & $\pm 28,27$ & 18,18 & $\pm 16,83$ & 21,66 & $\pm 21,86$ & 16,97 & $\pm 14,81$ & 24 \\
\hline recheado, doces ou & Sem EAAB & 17,97 & $\pm 31,69$ & 32,48 & $\pm 34,39$ & 16,85 & $\pm 31,35$ & 15,69 & $\pm 22,99$ & 50 \\
\hline guloseimas & valor de $\mathrm{p}$ & & & & & & & & & \\
\hline
\end{tabular}

Resultados apresentados a partir de percentual médio (\% médio) dos indicadores e desvio padrão (DP)

AME: Aleitamento Materno Exclusivo; AMC: Aleitamento Materno Complementado; EAAB: Estratégia Amamenta Alimenta Brasil

* Teste de Mann-Whitney comparando as Unidades de Saúde (USs) com e sem implantação da EAAB

** valor de p significativo $(<0,05)$

Fonte: Autores.

\section{Discussão}

No presente estudo, foi possível identificar que $100 \%$ das equipes das USs nas oficinas de implantação da EAAB, contaram com a participação de $80-85 \%$ dos profissionais (critérios 1). Os resultados também apontaram que os indicadores de promoção e manutenção do AM e ACS na primeira infância foram mais positivos nas USs com EAAB.

Todas as USs com a EAAB comprometeram-se com ações sistemáticas (individuais e coletivas), para a promoção do AM e ACS (critério 2). Dentre as ações propostas e avaliadas, a maioria foi implementada total ou parcialmente pelas equipes durante as consultas médicas, de enfermagem, odontológicas, nos grupos de educação em saúde e visitas domiciliares, impactando positivamente na continuidade do AME, AMC e ACS. Os impactos de ações e/ou intervenções de educação em saúde no aleitamento materno foram identificados em estudo realizado por Huang et al. (2019), no qual a partir de dois grupos- intervenção e controle - os autores identificaram que as mulheres que participaram de ações individualizads de apoio ao aleitamento materno durante o acompanhamento pré-natal e pós parto melhoraram significativamente as taxas de adesão ao aleitamento materno do nascimento até o quarto mês de vida do bebê. De acordo com Lopes et al. (2018) cabe aos profissionais de saúde o aconselhamento e incentivo de práticas alimentares adequadas, destacando-se a importância da participação da equipe na adesão e execução dessas ações. Esse incentivo por parte dos profissionais também foi evidenciado por Huang et al. (2019), uma vez que os autores evidenciaram que o apoio dos profissionais de saúde reduziu significativamente o uso de fórmulas e da mamadeira (Huang et al., 2019). Trazendo para a realidade das unidades com a $\mathrm{EAAB}$, os dados referentes ao aleitamento materno corroboram com as situações supracitadas, uma vez que em estudo que comparou a prevalência de crianças em AME em estabelecimentos privados e públicos, sem e com certificação na Rede Amamenta Brasil (estratégia anterior a EAAB), Passanha et al. (2013) mostraram que as chances de AM são maiores quando 
as crianças são assistidas por estabelecimentos certificados na estratégia, como observado no presente estudo. Este fato reforça a importância de políticas e estratégias que apoiem as ações das equipes de saúde para a promoção do AME, AMC e ACS.

Em relação ao preenchimento do marcador de consumo alimentar (critério 3), este vêm sendo realizado parcialmente por algumas USs, principalmente para crianças maiores de um ano, podendo ser justificado pelo aumento nos intervalos das consultas de rotina. Dentre algumas dificuldades de preenchimento, observou-se ausência de comunicação e dificuldades de alguns membros das USs na rotina do preenchimento. A implantação das ações pactuada na EAAB depende diretamente da receptividade da equipe e dos gestores locais, inclusive no sentido de incentivar o sistema de informação em saúde, com o preenchimento do banco de dados de indicadores de consumo alimentar (Bortolino, 2017). Apesar deste preenchimento parcial, observou-se melhores resultados dos indicadores relacionados à AM e ACS. O preenchimento adequado dos marcadores é importante, pois, auxilia na orientação de ações de atenção integral à saúde e na promoção do perfil alimentar e nutricional da população assistida (Brasil, 2015b).

Ao avaliar o desenvolvimento de ações de incentivo ao AM e ACS (critério 4), as USs mostraram-se interessadas em monitorar seus indicadores, criando instrumentos próprios, que permitem reavaliar resultados e planejar novas ações, quando necessário. Em estudo de avaliação do grau de implantação da Rede Amamenta Brasil, Venâncio et al. (2016), demonstraram que quanto maior o número de critérios que uma US cumpre em relação à estratégia, maiores são os índices de AM. Observouse no presente estudo que todas as USs propuseram ações durante a oficina, colocando-as em prática durante o período de monitoramento, podendo ser um motivo para a melhora dos indicadores.

Ao compararmos os percentuais médios dos indicadores de AM e ACS nas USs com e sem EAAB, evidenciou-se que os resultados relacionados ao AME e AMC foram melhores nas unidades com a EAAB. Esses resultados podem estar relacionados ao manejo ampliado da amamentação, demostrando que a capacitação e a informação dos profissionais das USs com a EAAB melhoram as ações de promoção, proteção e apoio ao AM. Além disso, a partir de 2017, o município intensificou as ações para mobilização social em prol do AM, como por exemplo, o "Agosto Dourado" (mês dedicado à promoção do AM) e a "Semana Municipal de Aleitamento Materno", integrando ações de suporte, incentivo e educação descentralizadas em toda a rede de atenção à saúde municipal. Segundo Carvalho e Tavares (2017), o manejo ampliado da amamentação inclui, além dos conhecimentos clínicos da lactação, conhecimentos necessários para que as equipes promovam, protejam e apoiem a amamentação na sua coletividade, família e comunidade, garantindo assim uma abordagem interdisciplinar e transversal que auxilia a mulher nos diversos aspectos envolvidos no processo de amamentar.

Vale ressaltar que essa melhora não foi imediata, sendo estatisticamente significativa no ano de 2018 (para AME) e nos anos de 2017/2018 (para AMC) e, por tratar-se de uma política recente, a literatura ainda traz dados incipientes destes indicadores para comparação. Apesar do percentual médio de AME encontrado estar abaixo do preconizado pela OMS, os dados mostraram melhora neste indicador nas USs estudadas, entretanto, para confirmar o avanço nos indicadores recomendase que sejam realizados estudos longitudinais e que esforços continuem existindo nesta direção.

O consumo de bebidas adoçadas não apresentou diferenças estatisticamente significativas entre as USs com e sem EAAB, indo ao encontro do estudo de Longo-Silva et al. (2015), onde a cultura alimentar da família e da sociedade, a acessibilidade à alimentos de qualidade e a influência da mídia sobre os hábitos alimentares, podem interferir diretamente no consumo dessas bebidas ultra processadas. O consumo de macarrão instantâneo, salgadinhos de pacote ou biscoitos salgados apresentou-se maior nas unidades sem a EAAB, ao passo que o consumo de biscoitos recheados, doces ou guloseimas também apresentou-se maior nas USs sem EAAB até o ano de 2017, quando o indicador apresentou diferença significativa, passando o consumo a ser maior nas unidades com a EAAB.

O consumo de alimentos no Brasil pôde ser identificado a partir da Pesquisa de Orçamentos Familiares (IBGE, 2010), indicou diminuição do consumo de alimentos básicos, como arroz e feijão e aumento do consumo de alimentos processados e 
ultraprocessados, como macarrão instantâneo, refrigerantes, biscoitos, entre outros (Brasil, 2015a). Em estudo realizado com crianças de zero a 36 meses de creches públicas, o consumo de suco industrializado antes do primeiro ano de vida esteve presente em mais da metade dos participantes, sendo que cerca de $10 \%$ o fizeram antes dos 6 meses, evidenciando a introdução de alimentos de forma inoportuna e precoce (Longo et al., 2015). Estes indicadores mostram a necessidade de adoção de novas ações por parte do município, principalmente no que diz respeito à educação continuada para as equipes, com o intuito redirecionar as ações para a redução do consumo de alimentos ultraprocessados. Além disso, faz-se necessário um maior tempo de estudo para que os efeitos da EAAB nestes indicadores possam ser observados.

Em estudo sobre a infância e alimentação infantil, Hotz e Gibson (2005) apontaram que o crescimento e desenvolvimento podem ter mudanças positivas quando sofrem intervenção efetiva de práticas alimentares adequadas e saudáveis, em particular nos países em desenvolvimento. Em Piracicaba, o número de USs com a estratégia implantada, no período estudado, era de 26,76\% ao passo que o índice nacional, no período de 2013 a 2016, apresentou 9,42\% das USs com a EAAB (Passanha et al., 2013). Sendo assim, para que a implantação da EAAB em nível nacional torne-se mais efetiva, é necessário que os gestores façam um diagnóstico da atual situação, a fim de apoiar os Estados e Municípios neste processo (Carvalho \& Tavares, 2017). Em estudo de avaliação da Rede Amamenta Brasil, Venâncio et al. (2013) identificaram que uma gestão municipal sensibilizada e atuante, com coordenador municipal da Estratégia e tutores atuantes vinculados a um projeto institucional, são fundamentais para o sucesso na implantação da Rede.

Uma das dificuldades encontradas foi a alta rotatividade dos profissionais nas USs, dificultando o cumprimento dos critérios da $\mathrm{EAAB}$ e, consequentemente, interferindo na capacitação das equipes de maneira integral e na avaliação da implantação da EAAB. Além disso, a avaliação do cumprimento das ações pactuadas nas oficinas de implantação foi realizada com base no relato das equipes, obtidos a partir do instumento aplicado. Apesar destas limitações, a EAAB tornou-se uma importante ferramenta de mudança no processo de trabalho das equipes no município, demonstrando uma maior segurança e uniformização para desenvolver ações de apoio ao AM e ACS, sendo possível contribuir para o aumento da prevalência desses indicadores.

\section{Conclusão}

Houve boa adesão à EAAB por parte dos membros das equipes das US e os indicadores de promoção e manutenção dos AM e ACS foram melhores nas unidades que participaram da EAAB. Mais estudos sobre a implantação dessa estratégia são necessários em outros municípios, garantindo assim a continuidade e aprimoramento das ações propostas no âmbito da Atenção Primária à Saúde.

\section{Agradecimentos}

À Secretaria Municipal de Saúde, ao Departamento de Atenção Básica do Município e às Unidades de Saúde, por permitirem a realização deste trabalho, pelo fornecimento dos dados e apoio durante a coleta dos dados.

\section{Referências}

Alves, J. D. S., Oliveira, M. I. C. D., \& Rito, R. V. V. F. (2018). Orientações sobre amamentação na atenção básica de saúde e associação com o aleitamento materno exclusivo. Ciência \& Saúde Coletiva, 23, 1077-1088.

Arreaza, A. L. V., \& Moraes, J. C. D. (2010). Contribuição teórico-conceitual para a pesquisa avaliativa no contexto de vigilância da saúde. Ciência \& Saúde Coletiva, 15, 2627-2638.

Bortolini, G. A. (2017). Avaliação da implementação da Estratégia Amamenta e aAimenta Brasil (EAAB). 
Brasil. Ministério da Saúde (2013b). Portaria ${ }^{\circ}$ 1.920, de 5 de setembro de 2013. Institui a Estratégia Nacional para Promoção do Aleitamento Materno e Alimentação Complementar Saudável no Sistema Único de Saúde (SUS)-estratégia Amamenta e Alimenta Brasil. http://bvsms.saude.gov. br/bvs/saudelegis/gm/2013/prt1920_05_09_2013.html

Brasil. Ministério da Saúde. Secretaria de Atenção à Saúde. (2015). Estratégia Nacional para Promoção do Aleitamento Materno e Alimentação Complementar Saudável no Sistema Único de Saúde: Manual de implementação. Brasília: Ministério da Saúde. https://bvsms.saude.gov.br/bvs/p ublicacoes/estrategia_nacional_promocao_aleitamento_materno.pdf

Brasil. Ministério da Saúde. Secretaria de Atenção à Saúde. Departamento de Ações Programáticas Estratégicas. (2013). Análise de implantação da Rede Amamenta Brasil: Relatório de pesquisa. Brasília: Ministério da Saúde. https://bvsms.saude.gov.br/bvs/publicaco es/analise_implantacao_amamenta_brasil_relatorio.pdf

Brasil. Ministério da Saúde. Secretaria de Atenção à Saúde. Departamento de Ações Programáticas Estratégicas. (2015). Saúde da criança: aleitamento materno e alimentação complementar. Brasília: Ministério da Saúde. http://bvsms.saude.gov.br/bvs/publicacoes/saude_c rianca_aleitamento_materno_cab23.pdf

Brasil. Ministério da Saúde. Secretaria de Atenção à Saúde. Departamento de Ações Programáticas Estratégicas. (2009). Estratégicas. II Pesquisa de prevalência de Aleitamento Materno nas Capitais Brasileiras e Distrito Federal. Brasília: Ministério da Saúde. http://bvsms.saude.gov.b r/bvs/publicacoes/pesquisa_prevalencia_aleitamento_materno.pdf

Brasil. Ministério da Saúde. Secretaria de Atenção à Saúde. Departamento de Atenção Básica. (2015). Orientações para avaliação de marcadores de consumo alimentar na atenção básica. http://bvsms.saude.gov.br/bvs/publicacoes/marcadores_consumo_alimentar_atencao_basica.pdf

Carvalho, M. R. C, Tavares, L. A. M.. (2017). Amamentação: bases científicas. (4a ed.), Guanabara Koogan.

Cunha, A. J. L. A. D., Leite, Á. J. M., \& Almeida, I. S. D. (2015). Atuação do pediatra nos primeiros mil dias da criança: a busca pela nutrição e desenvolvimento saudáveisłr. Jornal de Pediatria, 91, S44-S51.

Hotz, C., \& Gibson, R. S. (2005). Participatory nutrition education and adoption of new feeding practices are associated with improved adequacy of complementary diets among rural Malawian children: a pilot study. European journal of clinical nutrition, 59(2), 226-237.

Huang P, Yao J, Liu X, \& Luo B (2019). Individualized intervention to improve rates of exclusive breastfeeding. Medicine. 98:47.

Instituto Brasileiro de Geografia e Estatística (IBGE) (2010). Pesquisa de orçamentos familiares 2008-2009: despesas, rendimentos e condições de vida. em https://biblioteca.ibge.gov.br/visualizacao/livros/liv45130.pdf

Instituto Brasileiro de Geografia e Estatística (IBGE) (2017). Cidades. https://cidades.ibge.gov.br/brasil/sp/piracicaba/panorama

Instituto de Pesquisas e Planejamento de Piracicaba (IPPLAP) (2017). Equipamentos e serviços municipais de saúde de Piracicaba - 2017. http://www.ipplap.com.br/docs/Equipamentos\%20e\%20Servicos\%20Municipais\%20de\%20Saude\%20-\%202017.pdf

Jaime, P. C., Silva, A. C. F. D., Lima, A. M. C. D., \& Bortolini, G. A. (2011). Ações de alimentação e nutrição na atenção básica: a experiência de organização no Governo Brasileiro. Revista de Nutrição, 24, 809-824.

Longo-Silva, G., Silveira, J. A. C., Menezes, R. C. E. D., \& Toloni, M. H. D. A. (2017). Age at introduction of ultra-processed food among preschool children attending day-care centers. Jornal de pediatria, 93, 508-516.

Lopes, W. C., Marques, F. K. S., Oliveira, C. F. D., Rodrigues, J. A., Silveira, M. F., Caldeira, A. P., \& Pinho, L. D. (2018). Alimentação de crianças nos primeiros dois anos de vida. Revista Paulista de Pediatria, 36, 164-170.

Passanha, A., Benício, M. H. D. A., Venâncio, S. I., \& Reis, M. C. G. D. (2013). Implantação da Rede Amamenta Brasil e prevalência de aleitamento materno exclusivo. Revista de Saúde Pública, 47, 1141-1148.

Silveira, P. P., Portella, A. K., Goldani, M. Z., \& Barbieri, M. A. (2007). Origens desenvolvimentistas da saúde e da doença (DOHaD). Jornal de Pediatria, 83, 494-504.

Torigoe, C. Y., Asakura, L., Sachs, A., Silva, C. V. D. D., Abrão, A. C. F. D. V., Santos, G. M. S. D., \& Coelho, L. D. C. (2012). Influence of the nutritional intervention in complementary feeding practices in infants. Journal of Human Growth and Development, 22(1), 85-92.

Venancio, S. I., Giugliani, E. R. J., Silva, O. L. D. O., Stefanello, J., Benicio, M. H. D. A., Reis, M. C. G. D., Issler, R. M. S., Santo, L. C. E., Cardoso, M. R. A., \& Rios, G. S. (2016). Associação entre o grau de implantação da Rede Amamenta Brasil e indicadores de amamentação. Cadernos de Saúde Pública, 32, $\mathrm{e} 00010315$.

Venancio, S. I., Saldiva, S. R. D. M., \& Monteiro, C. A. (2013). Tendência secular da amamentação no Brasil. Revista de Saúde Pública, 47, $1205-1208$.

Victora, C. G., Horta, B. L., De Mola, C. L., Quevedo, L., Pinheiro, R. T., Gigante, D. P., Gonlaçves, H., \& Barros, F. C. (2015). Association between breastfeeding and intelligence, educational attainment, and income at 30 years of age: a prospective birth cohort study from Brazil. The lancet global health, 3(4), e199-e205.

Victora, C. G., Bahl, R., Barros, A. J., França, G. V., Bahl, R., Rollins, N. C., Horton, S., Krasevec, J., Murch, S., Sankar, M. J., \& Walker N (2016). Amamentação no século 21: epidemiologia, mecanismos e efeitos ao longo da vida. The Lancet , 387 (10017), 475-490.

Walters, D. D., Phan, L., \& Mathisen, R. (2019). The cost of not breastfeeding: global results from a new tool. Health policy and planning, 34(6), 407-417.

World Health Organization. United Nations Children's Fundation. (2017). Nurturing the health and wealth of nations: the investment case for breastfeeding Global breastfeeding collective - executive summary. United Nations Children's Fundation https://www.who.int/nutrition/publications/infantfeeding/global-bfcollective-investmentcase/en/ 